\title{
Causal networks in the rat barrel cortex provide a signature of stimulus encoding
}

\author{
Seif Eldawlatly ${ }^{1 *}$, Karim Oweiss ${ }^{1,2}$ \\ From Nineteenth Annual Computational Neuroscience Meeting: CNS*2010 \\ San Antonio, TX, USA. 24-30 July 2010
}

Identifying stimulus-dependent cortical circuits is a fundamental goal in systems neuroscience. Graph-theoretic techniques provide an elegant tool to infer such circuitry. Recently, we have proposed to use Dynamic Bayesian Networks (DBN) as an efficient graphical model that explains the observed spike train data in local cortical circuits. A unique advantage of DBNs over other metrics of neuronal connectivity (such as crosscorrelograms) is its ability to explain the temporally precise activity of an observed neuron taking into account the activity of the entire observed population.

We recorded simultaneously the ensemble neural activity using 32-channel microelectrode arrays from Layer $\mathrm{V}$ of the primary somatosensory (barrel) cortex in 3 anesthetized rats. An average population size of $20 \pm 7$ single units/rat was identified. Responses were recorded during stimulation of individual whiskers (3 whiskers/ rat). Each whisker was horizontally deflected with a displacement of $80 \mu \mathrm{m}$ for $100 \mathrm{~ms}$ at $1 \mathrm{~Hz}$ frequency. Spike trains within $100 \mathrm{~ms}$ of stimulus onset were analyzed using DBN. To rule out the existence of spurious connections inferred by co-modulation of firing rates, a jittered version of the spike trains was formed in which each spike was randomly jittered in a uniform interval of $[-5,5] \mathrm{ms}$. Common connections inferred in the original and jittered data were eliminated.

Results demonstrate that stable stimulus-driven network states were obtained, with $22 \%$ of the inferred connections appearing in more than $35 \%$ of the trials. To examine the validity of these results, we computed the correlation between the response latency of each neuron and the number of inferred pre-synaptic/post-synaptic connections for each stimulated whisker. In a causal network context, we hypothesized that pre-synaptic neurons that fire before other post-synaptic neurons in the observed population should have shorter response latency reminiscent of a "signaling mechanism" to other neurons about stimulus presence. Therefore, these neurons were expected to have more post-synaptic connections than their large response latency counterparts. Correlation between the response latency and the number of post-synaptic (pre-synaptic) connections was $-0.63(+0.61)$ consistent with our hypothesis. Finally, we used $90 \%$ of the trials to obtain a template network model for each whisker stimulated. We then decoded the spike trains in the remaining $10 \%$ of the trials to determine the corresponding whisker identity. A decoding accuracy of $97 \%$ was obtained indicating that the obtained causal networks are stimulus-dependent in which the population acts cooperatively rather than independently to encode the underlying stimulus.

\section{Acknowledgement}

This work was supported by NIH grants \#NS054148 and NS062031.

\section{Author details}

${ }^{1}$ Electrical and Computer Engineering Dept., Michigan State University, East Lansing, MI 48824, USA. ${ }^{2}$ Neuroscience Program, Michigan State University, East Lansing, MI 48824, USA.

Published: 20 July 2010

doi:10.1186/1471-2202-11-S1-018

Cite this article as: Eldawlatly and Oweiss: Causal networks in the rat

barrel cortex provide a signature of stimulus encoding. BMC

Neuroscience 2010 11(Suppl 1):018.

* Correspondence: eldawlat@egr.msu.edu

${ }^{1}$ Electrical and Computer Engineering Dept., Michigan State University, East Lansing, Ml 48824, USA 